\title{
A new extension of Srivastava's triple hypergeometric functions and their associated properties
}

https://doi.org/10.1515/anly-2020-0036

Received July 28, 2020; revised August 20, 2020; accepted August 20, 2020

\begin{abstract}
In this paper, we define a new extension of Srivastava's triple hypergeometric functions by using a new extension of Pochhammer's symbol that was recently proposed by Srivastava, Rahman and Nisar [H. M. Srivastava, G. Rahman and K. S. Nisar, Some extensions of the Pochhammer symbol and the associated hypergeometric functions, Iran. J. Sci. Technol. Trans. A Sci. 43 (2019), no. 5, 2601-2606]. We present their certain basic properties such as integral representations, derivative formulas, and recurrence relations. Also, certain new special cases have been identified and some known results are recovered from main results.
\end{abstract}

Keywords: Pochhammer's symbols, Gamma function, hypergeometric functions, Bessel and modified Bessel functions, Appell functions, Whittaker function, Srivastava's triple hypergeometric functions

MSC 2010: 11B83, 11S80, 05A19

\section{Introduction}

In recent years, many researchers have presented and investigated various extensions of the well-known special functions. We refer the interested readers to the works $[1,2,6-8,11,13,14,16,27,30]$. In 1994, Chaudhry and Zubair [3] (see also [4]) have proposed an extension of the classical gamma function $\Gamma(z)$ (see [24]) by employing the regularization term $\exp \left(-\frac{p}{t}\right)$ as follows:

$$
\Gamma_{p}(\zeta)= \begin{cases}\int_{0}^{\infty} \theta^{\zeta-1} \exp \left(-\theta-\frac{p}{\theta}\right) \mathrm{d} \theta & (\mathbb{R}(p)>0, \zeta \in \mathbb{C}), \\ \Gamma(\zeta) & (p=0, \mathbb{R}(\zeta)>0) .\end{cases}
$$

Throughout this paper, we denote the sets of positives integers, negative integers and complex numbers by $\mathbb{N}, \mathbb{Z}^{-}$and $\mathbb{C}$, respectively and we let $\mathbb{N}_{0}=\mathbb{N} \cup\{0\}$ and $\mathbb{Z}_{0}^{-}=\mathbb{Z}^{-} \cup\{0\}$.

In [28], Srivastava, Parmar and Joshi proposed the extension of three Lauricella functions $F_{A}^{(n)}, F_{B}^{(n)}$ and $F_{C}^{(n)}$ of $n$ variables which are the generalized form of the corresponding Appell functions $F_{2}, F_{4}$ and $F_{3}$, respectively.

\footnotetext{
*Corresponding author: Serkan Araci, Department of Economics, Faculty of Economics, Administrative and Social Sciences, Hasan Kalyoncu University, TR-27410 Gaziantep, Turkey, e-mail: serkan.araci@hku.edu.tr. https://orcid.org/0000-0002-3950-6864

Abdus Saboor, Zunaira Anjum, Institute of Numerical Sciences, Kohat University of Science \& Technology, Kohat 26000, Pakistan, e-mail: dr.abdussaboor@kust.edu.pk, anjum499499@gmail.com

Gauhar Rahman, Department of Mathematics, Shaheed Benazir Bhutto University, Sheringal, Upper Dir, Pakistan, e-mail: gauhar55uom@gmail.com Kottakkaran Sooppy Nisar, Department of Mathematics, Prince Sattam bin Abdulaziz University, Wadi Aldawaser, 11991, Saudi Arabia, e-mail: n.sooppy@psau.edu.sa
} 
Recall the following extension of the second Appell function $F_{2}$ (see [28]) and the two confluent Appell series [25] defined respectively by

$$
F_{2}\left[(\sigma, p), b_{1}, b_{2} ; c_{1}, c_{2} ; \theta_{1}, \theta_{2}\right]=\frac{\sum_{m_{1}, m_{2}=0}^{\infty}(\sigma, p)_{m_{1}+m_{2}}\left(b_{1}\right)_{m_{1}}\left(b_{2}\right)_{m_{2}}}{\left(c_{1}\right)_{m_{1}}\left(c_{2}\right)_{m_{2}}} \frac{\theta_{1}^{m_{1}}}{m_{1} !} \frac{\theta_{2}^{m_{1}}}{m_{2} !},
$$

where $\mathbb{R}(p) \geq 0,\left|\theta_{1}\right|+\left|\theta_{2}\right|<1$ when $p=0$, and

$$
\psi_{2}\left[\sigma ; c_{1}, c_{2} ; \theta_{1}, \theta_{2}\right]=\sum_{m, n=0}^{\infty} \frac{(\sigma)_{m+n}}{\left(c_{1}\right)_{m}\left(c_{2}\right)_{n}} \frac{\theta_{1}^{m}}{m !} \frac{\theta_{2}^{n}}{n !} \quad\left(\left|\theta_{1}\right|<\infty,\left|\theta_{2}\right|<\infty\right)
$$

and

$$
\phi_{3}\left[\sigma ; c ; \theta_{1}, \theta_{2}\right]=\sum_{m, n=0}^{\infty} \frac{(\sigma)_{m}}{(c)_{m+n}} \frac{\theta_{1}^{m}}{m !} \frac{\theta_{2}^{n}}{n !} \quad\left(\left|\theta_{1}\right|<\infty,\left|\theta_{2}\right|<\infty\right) .
$$

In [29], Srivastava, Rahman and Nisar recently defined the following extension of the generalized hypergeometric function:

$$
{ }_{p} F_{q}\left[\left(\eta_{1} ; p, v\right), \ldots,\left(\eta_{r}\right) ;\left(\varrho_{1}^{\prime}\right), \ldots,\left(\varrho_{s}^{\prime}\right) ; z\right]=\sum_{m=0}^{\infty} \frac{\left(\eta_{1} ; p, v\right)_{m} \cdots\left(\eta_{r}\right)_{m}}{\left(\varrho_{1}^{\prime}\right)_{m} \cdots\left(\varrho_{s}^{\prime}\right)_{m}} \frac{z^{m}}{m !},
$$

where $\eta_{i} \in \mathbb{C}$ for $i=1,2, \ldots, r, \varrho_{j}^{\prime} \in \mathbb{C}$ for $j=1,2, \ldots, s, \varrho_{j}^{\prime} \neq 0,-1,-2, \ldots$, and $(\varrho ; p, v)_{\mu}$ is the new extension of the generalized Pochhammer symbol defined by (see [29])

$$
(\varrho ; p, v)_{\mu}= \begin{cases}\frac{\Gamma_{v}(\varrho+\mu ; p)}{\Gamma(\varrho)}, & (\mathbb{R}(p), \mathbb{R}(v)>0, \varrho, \mu \in \mathbb{C}), \\ (\varrho ; p)_{\mu} & (v=0, \varrho, \mu \in \mathbb{C} \backslash\{0\}) .\end{cases}
$$

The integral representation of $(\varrho ; \sigma, v)_{\mu}$ is defined by

$$
(\varrho ; p, v)_{\mu}=\sqrt{\frac{2 p}{\pi}} \frac{1}{\Gamma(\varrho)} \int_{0}^{\infty} t^{\varrho+\mu-\frac{3}{2}} e^{-t} K_{v+\frac{1}{2}}\left(\frac{p}{t}\right) \mathrm{d} t .
$$

where $K_{v}(\cdot)$ is the well-known modified Bessel function of order $v$. When we consider $v=0$ in (1.4), by substituting the result

$$
K_{\frac{1}{2}}(z)=\sqrt{\frac{\pi}{2 z}} e^{-z}
$$

we get the following formula (see [23]):

$$
(\varrho ; p, 0)_{\mu}=(\varrho ; p)_{\mu}=\frac{1}{\Gamma(\varrho)} \int_{0}^{\infty} t^{\varrho+\mu-1} e^{-t} \mathrm{~d} t .
$$

Motivated by the above investigations, we propose a new extension of the generalized Srivastava's triple hypergeometric functions $H_{A}, H_{B}$ and $H_{C}$ (see [5]) and investigate the various basic properties of the said functions.

\section{Extension of the generalized Srivastava's triple hypergeometric functions}

The following new extension of the generalized Srivastava's triple hypergeometric functions $H_{A}, H_{B}$ and $H_{C}$ (see [5]) in terms of the new extended Pochhammer's symbol in (1.3) recently proposed by Srivastava et al. [29] are presented in this section:

$$
H_{A}\left[(\sigma ; p, v), b_{1}, b_{2} ; c_{1}, c_{2} ; \theta_{1}, \theta_{2}, \theta_{3}\right]=\frac{\left.\sum_{l_{1}, l_{2}, l_{3}=0}^{\infty}(\sigma ; p, v)_{l_{1}+l_{3}}\left(b_{1}\right)_{l_{1}+l_{2}} b_{2}\right) l_{2}+l_{3}}{\left(c_{1}\right)_{l}\left(c_{2}\right) l_{2}+l_{3}} \frac{\theta_{1}^{l_{1}}}{l_{1} !} \frac{\theta_{2}^{l_{2}}}{l_{2} !} \frac{\theta_{3}^{l_{3}}}{l_{3} !},
$$


where $p \geq 0,\left|\theta_{1}\right|<r_{1},\left|\theta_{2}\right|<s_{1},\left|\theta_{3}\right|<t_{1}$ and $r_{1}+s_{1}+t_{1}=1+s_{1} t_{1}$,

$$
H_{B}\left[(\sigma ; p, v), b_{1}, b_{2} ; c_{1}, c_{2}, c_{3} ; \theta_{1}, \theta_{2}, \theta_{3}\right]=\frac{\sum_{l_{1}, l_{2}, l_{3}=0}^{\infty}(\sigma ; p, v)_{l_{1}+l_{3}}\left(b_{1}\right)_{l_{1}+l_{2}}\left(b_{2}\right) l_{2}+l_{3}}{\left.c_{1}\right)_{l}\left(c_{2}\right)_{m}\left(c_{3}\right)_{l_{3}}} \frac{\theta_{1}^{l_{1}}}{l_{1} !} \frac{\theta_{2}^{l_{2}}}{l_{2} !} \frac{\theta_{3}^{l_{3}}}{l_{3} !},
$$

where $p \geq 0,\left|\theta_{1}\right|<r_{1},\left|\theta_{2}\right|<s_{1},\left|\theta_{3}\right|<t_{1}$ and $r_{1}+s_{1}+t_{1}+2 \sqrt{r_{1} s_{1} t_{1}}=1$, and

$$
H_{C}\left[(\sigma ; p, v), b_{1}, b_{2} ; \gamma ; \theta_{1}, \theta_{2}, \theta_{3}\right]=\frac{\sum_{l_{1}, l_{2}, l_{3}=0}^{\infty}(\sigma ; p, v)_{l_{1}+l_{3}}\left(b_{1}\right)_{l_{1}+l_{2}}\left(b_{2}\right) l_{2}+l_{3}}{(y)_{l_{1}+l_{2}+l_{3}}} \frac{\theta_{1}^{l_{1}}}{l_{1} !} \frac{\theta_{2}^{l_{2}}}{l_{2} !} \frac{\theta_{3}^{l_{3}}}{l_{3} !},
$$

where $p \geq 0,\left|\theta_{1}\right|<r_{1},\left|\theta_{2}\right|<s_{1},\left|\theta_{3}\right|<t_{1}, r_{1}+s_{1}+t_{1}-2 \sqrt{\left(1-r_{1}\right)\left(1-s_{1}\right)\left(1-t_{1}\right)}<2$ and $\sigma, b_{1}, b_{2} \in \mathbb{C}$ and $\gamma, c_{1}, c_{2}, c_{3} \in \mathbb{C} \backslash\left\{\mathbb{Z}_{0}^{-}\right\}$.

Remark 2.1. If we let $v=0$ in (2.1)-(2.3), then we get the generalized Srivastava's triple hypergeometric functions $H_{A}, H_{B}$ and $H_{C}$ proposed earlier by Choi and Parmar [5], respectively.

Remark 2.2. When setting $\theta_{2}=0$ in (2.1) and (2.2), we get the following new extension of the second Appell hypergeometric function $F_{2}$ defined by

$$
F_{2}\left[(\sigma ; p, v), b_{1}, b_{2} ; c_{1}, c_{2} ; \theta_{1}, \theta_{3}\right]=\frac{\sum_{l_{1}, l_{2}=0}^{\infty}(\sigma ; p, v)_{l_{1}+l_{2}}\left(b_{1}\right)_{l}\left(b_{2}\right)_{l_{2}}}{\left(c_{1}\right)_{l}\left(c_{2}\right)_{l_{2}}} \frac{\theta_{1}^{l_{1}}}{l_{1} !} \frac{\theta_{2}^{l_{2}}}{l_{2} !}
$$

where $\mathbb{R}(p) \geq 0,\left|\theta_{1}\right|+\left|\theta_{2}\right|<1$ when $p=0$.

Similarly, when we set $\theta_{2}=0$, we get the following new extension of the first Appell hypergeometric function $F_{1}$ defined by

$$
F_{1}\left[(\sigma ; p, v), b_{1}, b_{2} ; \gamma ; \theta_{1}, \theta_{2}\right]=\frac{\sum_{l_{1}, l_{2}=0}^{\infty}(\sigma ; p, v)_{l_{1}+l_{2}}\left(b_{1}\right)_{l}\left(b_{2}\right) l_{l_{2}}}{(c)_{l_{1}+l_{2}}} \frac{\theta_{1}^{l_{1}}}{l_{1} !} \frac{\theta_{2}^{l_{2}}}{l_{2} !},
$$

where $\mathbb{R}(p) \geq 0,\left|\theta_{1}\right|<1,\left|\theta_{2}\right|<1$ when $p=0$.

\section{Integral representations}

Certain various integral representations of the newly defined extension of Srivastava's triple hypergeometric functions (2.1), (2.2) and (2.3) are presented in this section. An integral representation of Srivastava's hypergeometric function $H_{A}$ in (2.1) is presented in the following theorem.

Theorem 3.1. For $\mathbb{R}(p)>0, \max \left\{\mathbb{R}\left(\theta_{2}\right), \mathbb{R}\left(\theta_{3}\right)\right\}<1$ and $\min \left\{\mathbb{R}(\sigma), \mathbb{R}\left(b_{1}\right)\right\}>0$, the integral

$$
\begin{array}{r}
H_{A}\left[(\sigma ; p, v), b_{1}, b_{2} ; c_{1}, c_{2} ; \theta_{1}, \theta_{2}, \theta_{3}\right]=\frac{\sqrt{\frac{2 p}{\pi}}}{\Gamma(\sigma) \Gamma\left(b_{1}\right)} \int_{0}^{\infty} \int_{0}^{\infty} t^{\sigma+l_{1}+l_{3}-\frac{3}{2}} s^{b_{1}+l_{1}+l_{2}-1} e^{-(t+s)} K_{v+\frac{1}{2}}\left(\frac{p}{t}\right) \\
\times{ }_{0} F_{1}\left(-; c_{1} ; \theta_{1} s t\right){ }_{1} F_{1}\left(b_{2}, ; c_{2} ; \theta_{2} s+\theta_{3} t\right) \mathrm{d} s \mathrm{~d} t
\end{array}
$$

holds for the hypergeometric function $H_{A}$ defined in (2.1).

Proof. We have

$$
(\sigma ; p, v))_{l_{1}+l_{3}}=\sqrt{\frac{2 p}{\pi}} \frac{1}{\Gamma(\sigma)} \int_{0}^{\infty} t^{\sigma+l_{1}+l_{3}-\frac{3}{2}} e^{-t} K_{v+\frac{1}{2}}\left(\frac{p}{t}\right) \mathrm{d} t
$$

and

$$
\left(b_{1}\right) l_{1}+l_{2}=\frac{1}{\Gamma\left(b_{1}\right)} \int_{0}^{\infty} s^{b_{1}+l_{1}+l_{2}-1} e^{-s} \mathrm{~d} s
$$


Using these both equations in (2.1), we obtain

$$
\begin{aligned}
& H_{A}\left[(\sigma ; p, v), b_{1}, b_{2} ; c_{1}, c_{2} ; \theta_{1}, \theta_{2}, \theta_{3}\right] \\
& \quad=\frac{\sum_{l_{1}, l_{2}, l_{3}=0}^{\infty} \sqrt{\frac{2 p}{\pi}} \frac{1}{\Gamma(\sigma)} \int_{0}^{\infty} t^{\sigma+l_{1}+l_{3}-\frac{3}{2}} e^{-t} K_{v+\frac{1}{2}}\left(\frac{p}{t}\right) \mathrm{d} t \frac{1}{\Gamma\left(b_{1}\right)} \int_{0}^{\infty} s^{b_{1}+l_{1}+l_{2}-1} e^{-s} \mathrm{~d} s\left(b_{2}\right)_{l_{2}+l_{3}} \frac{\theta_{1}^{l_{1}}}{l_{1} !} \frac{\theta_{2}^{l_{2}}}{l_{2} !} \frac{\theta_{3}^{l_{3}}}{l_{3} !}}{}=\sqrt{\frac{2 p}{\pi}} \frac{1}{\Gamma(\sigma)} \int_{0}^{\infty} t^{\sigma+l_{1}+l_{3}-\frac{3}{2}} e^{-t} K_{v+\frac{1}{2}}\left(\frac{p}{t}\right) \mathrm{d} t \frac{1}{\Gamma\left(b_{1}\right)} \int_{0}^{\infty} s^{b_{1}+l_{1}+l_{2}-1} e^{-s} \mathrm{~d} s \sum_{l_{1}, l_{2}, l_{3}=0}^{\infty} \frac{\left(b_{2}\right)_{l_{2}+l_{3}}}{\left(c_{1}\right)_{l}\left(c_{2}\right)_{l_{2}+l_{3}}} \frac{\theta_{1}^{l_{1}}}{l_{1} !} \frac{\theta_{2}^{l_{2}}}{l_{2} !} \frac{\theta_{3}^{l_{3}}}{l_{3} !} \\
&= \frac{\sqrt{\frac{2 p}{\pi}}}{\Gamma(\sigma) \Gamma\left(b_{1}\right)} \int_{0}^{\infty} \int_{0}^{\infty} t^{\sigma+l_{1}+l_{3}-\frac{3}{2}} s^{b_{1}+l_{1}+l_{2}-1} e^{-(s+t)} K_{v+\frac{1}{2}}\left(\frac{p}{t}\right) \mathrm{d} s \mathrm{~d} t \sum_{l_{1}, l_{2}, l_{3}=0}^{\infty} \frac{1}{\left(c_{1}\right)_{l}} \frac{\left(b_{2}\right)_{l_{2}+l_{3}}}{\left(c_{2}\right)_{l_{2}+l_{3}}} \frac{\theta_{1}^{l_{1}} \frac{\theta_{2}}{l_{1}} \frac{\theta_{2}}{l_{2}} \frac{\theta_{3}^{l_{3}}}{l_{3} !}}{}
\end{aligned}
$$

Since

$$
\sum_{l=0}^{\infty} \frac{1}{\left(c_{1}\right)} \frac{\theta_{1}^{l_{1}}}{l_{1} !}={ }_{0} F_{1}\left(-; c_{1} ; \theta_{1} s t\right)
$$

and

$$
\sum_{m, n=0}^{\infty} \frac{\left(b_{2}\right)_{l_{2}+l_{3}}}{\left(c_{2}\right)_{l_{2}+l_{3}}} \frac{\theta_{2}^{l_{2}}}{l_{2} !} \frac{\theta_{3}^{l_{3}}}{l_{3} !}={ }_{1} F_{1}\left(b_{2}, ; c_{2} ; \theta_{2} s+\theta_{3} t\right) .
$$

we obtain

$$
\begin{aligned}
& H_{A}\left[(\sigma ; p, v), b_{1}, b_{2} ; c_{1}, c_{2} ; \theta_{1}, \theta_{2}, \theta_{3}\right] \\
& \quad=\frac{\sqrt{\frac{2 p}{\pi}}}{\Gamma(\sigma) \Gamma\left(b_{1}\right)} \int_{0}^{\infty} \int_{0}^{\infty} t^{\sigma+l_{1}+l_{3}-\frac{3}{2}} s^{b_{1}+l_{1}+l_{2}-1} e^{-(t+s)} K_{v+\frac{1}{2}}\left(\frac{p}{t}\right){ }_{0} F_{1}\left(-; c_{1} ; \theta_{1} s t\right)_{1} F_{1}\left(b_{2}, ; c_{2} ; \theta_{2} s+\theta_{3} t\right) \mathrm{d} s \mathrm{~d} t,
\end{aligned}
$$

as desired.

Theorem 3.2. For $\mathbb{R}(p)>0, \mathbb{R}\left(\theta_{2}\right)<1, \mathbb{R}\left(\theta_{3}\right)<1$, $\mathbb{R}(\sigma)>0$ and $\mathbb{R}\left(b_{1}\right)>0$, the integral

$$
\begin{aligned}
H_{B}\left[(\sigma ; p, v), b_{1}, b_{2} ; c_{1}, c_{2} ; c_{3} ; \theta_{1}, \theta_{2}, \theta_{3}\right]= & \frac{\sqrt{\frac{2 p}{\pi}}}{\Gamma(\sigma) \Gamma\left(b_{1}\right)} \int_{0}^{\infty} \int_{0}^{\infty} t^{\sigma+l_{1}+l_{3}-\frac{3}{2}} s^{b_{1}+l_{1}+l_{2}-1} e^{-(t+s)} K_{v+\frac{1}{2}}\left(\frac{p}{t}\right) \\
& \times{ }_{0} F_{1}\left(-; c_{1} ; \theta_{1} s t\right) \psi_{2}\left(b_{2}, ; c_{2} ; c_{3} ; \theta_{2} s, \theta_{3} t\right) \mathrm{d} s \mathrm{~d} t
\end{aligned}
$$

holds for the Srivastava hypergeometric function $H_{B}$ defined in (2.2).

Proof. We have

$$
(\sigma ; p, v)_{l_{1}+l_{3}}=\sqrt{\frac{2 p}{\pi}} \frac{1}{\Gamma(\sigma)} \int_{0}^{\infty} t^{\sigma+l_{1}+l_{3}-\frac{3}{2}} e^{-t} K_{v+\frac{1}{2}}\left(\frac{p}{t}\right) \mathrm{d} t
$$

and

$$
\left(b_{1}\right)_{l_{1}+l_{2}}=\frac{1}{\Gamma\left(b_{1}\right)} \int_{0}^{\infty} s^{b_{1}+l_{1}+l_{2}-1} e^{-s} \mathrm{~d} s .
$$

Using these both equations in (2.2), we obtain

$$
\begin{aligned}
H_{B}\left[(\sigma ; p, v), b_{1}, b_{2} ; c_{1}, c_{2} ; c_{3} ; \theta_{1}, \theta_{2}, \theta_{3}\right] \\
=\frac{\sum_{l_{1}, l_{2}, l_{3}=0}^{\infty} \sqrt{\frac{2 p}{\pi}} \frac{1}{\Gamma(\sigma)} \int_{0}^{\infty} t^{\sigma+l_{1}+l_{3}-\frac{3}{2}} e^{-t} K_{v+\frac{1}{2}}\left(\frac{p}{t}\right) \mathrm{d} t \frac{1}{\Gamma\left(b_{1}\right)} \int_{0}^{\infty} s^{b_{1}+l_{1}+l_{2}-1} e^{-s} \mathrm{~d} s\left(b_{2}\right)_{l_{2}+l_{3}} \frac{\theta_{1}^{l_{1}}}{l_{1} !} \frac{\theta_{2}^{l_{2}}}{l_{2} !} \frac{\theta_{3}^{l_{3}}}{l_{3} !}}{=\sqrt{\frac{2 p}{\pi}} \frac{1}{\Gamma(\sigma)} \int_{0}^{\infty} t^{\sigma+l_{1}+l_{3}-\frac{3}{2}} e^{-t} K_{v+\frac{1}{2}}\left(\frac{p}{t}\right) \mathrm{d} t \frac{1}{\Gamma\left(b_{1}\right)} \int_{0}^{\infty} s^{b_{1}+l_{1}+l_{2}-1} e^{-s} \mathrm{~d} s \sum_{l_{1}, l_{2}, l_{3}=0}^{\infty} \frac{\left(b_{2}\right)_{l_{2}+l_{3}}}{\left(c_{1}\right)_{l}\left(c_{2}\right)_{l_{2}}\left(c_{3}\right)_{l_{3}}} \frac{\theta_{1}^{l_{1}}}{l_{1} !} \frac{\theta_{2}^{l_{2}}}{l_{2} !} \frac{\theta_{3}^{l_{3}}}{l_{3} !}} \\
=\frac{\sqrt{\frac{2 p}{\pi}}}{\Gamma(\sigma) \Gamma\left(b_{1}\right)} \int_{0}^{\infty} \int_{0}^{\infty} t^{\sigma+l_{1}+l_{3}-\frac{3}{2}} s^{b_{1}+l_{1}+l_{2}-1} e^{-(s+t)} K_{v+\frac{1}{2}}\left(\frac{p}{t}\right) \mathrm{d} s \mathrm{~d} t \sum_{l_{1}, l_{2}, l_{3}=0}^{\infty} \frac{1}{\left(c_{1}\right)} \frac{\left(b_{2}\right)_{l}+l_{3}}{\left(c_{2}\right)_{l_{2}}\left(c_{3}\right)_{l_{3}}} \frac{\theta_{1}^{l_{1}}}{l_{1} !} \frac{\theta_{2}^{l_{2}} l_{2} !}{l_{3}} \frac{\theta_{3}^{l_{3}}}{l_{3} !}
\end{aligned}
$$


Hence

$$
\begin{aligned}
& H_{B}\left[(\sigma ; p, v), b_{1}, b_{2} ; c_{1}, c_{2} ; c_{3} ; \theta_{1}, \theta_{2}, \theta_{3}\right] \\
& \quad=\frac{\sqrt{\frac{2 p}{\pi}}}{\Gamma(\sigma) \Gamma\left(b_{1}\right)} \int_{0}^{\infty} \int_{0}^{\infty} t^{\sigma+l_{1}+l_{3}-\frac{3}{2}} s^{b_{1}+l_{1}+l_{2}-1} e^{-(t+s)} K_{v+\frac{1}{2}}\left(\frac{p}{t}\right){ }_{0} F_{1}\left(-; c_{1} ; \theta_{1} s t\right) \psi_{2}\left(b_{2}, ; c_{2} ; c_{3} ; \theta_{2} s \theta_{3} t\right) \mathrm{d} s \mathrm{~d} t .
\end{aligned}
$$

Using the identity in (1.1), we obtain

$$
\psi_{2}\left[\sigma ; c_{1}, c_{2} ; \theta_{1}, \theta_{2}\right]=\sum_{m, n=0}^{\infty} \frac{(\sigma)_{l_{2}+l_{3}}}{\left(c_{1}\right)_{l_{2}}\left(c_{2}\right)_{l_{3}}} \frac{\theta_{2}^{l_{2}}}{l_{2} !} \frac{\theta_{3}^{l_{3}}}{l_{3} !} .
$$

Theorem 3.3. For $\mathbb{R}(p)>0$, and $\min \left\{\mathbb{R}(\sigma), \mathbb{R}\left(b_{1}\right), \mathbb{R}\left(b_{2}\right)\right\}>0$, the triple integral

$$
\begin{aligned}
& H_{B}\left[(\sigma ; p, v), b_{1}, b_{2} ; c_{1}, c_{2} ; c_{3} ; \theta_{1}, \theta_{2}, \theta_{3}\right] \\
& =\frac{\sqrt{\frac{2 p}{\pi}}}{\Gamma(\sigma) \Gamma\left(b_{1}\right) \Gamma\left(b_{2}\right)} \int_{0}^{\infty} \int_{0}^{\infty} \int_{0}^{\infty} t^{\sigma+l_{1}+l_{3}-\frac{3}{2}} s^{b_{1}+l_{1}+l_{2}-1} u^{b_{2}+l_{2}+l_{3}-1} \\
& \quad \times e^{-(s+t+u)} K_{v+\frac{1}{2}}\left(\frac{p}{t}\right)_{0} F_{1}\left(-; c_{1} ; \theta_{1} s t\right) \times{ }_{0} F_{1}\left(-; c_{2} ; \theta_{2} u s\right){ }_{0} F_{1}\left(-; c_{3} ; \theta_{3} u t\right) \mathrm{d} t \mathrm{~d} s \mathrm{~d} u
\end{aligned}
$$

holds for the Srivastava hypergeometric function $H_{B}$ defined in (2.2).

Proof. We have

$$
\begin{aligned}
(\sigma ; p, v)_{l_{1}+l_{3}} & =\sqrt{\frac{2 p}{\pi}} \frac{1}{\Gamma(\sigma)} \int_{0}^{\infty} t^{\sigma+l_{1}+l_{3}-\frac{3}{2}} e^{-t} K_{v+\frac{1}{2}}\left(\frac{p}{t}\right) \mathrm{d} t, \\
\left(b_{1}\right)_{l_{1}+l_{2}} & =\frac{1}{\Gamma\left(b_{1}\right)} \int_{0}^{\infty} s^{b_{1}+l_{1}+l_{2}-1} e^{-s} \mathrm{~d} s, \\
\left(b_{2}\right)_{l_{2}+l_{3}} & =\frac{1}{\Gamma\left(b_{2}\right)} \int_{0}^{\infty} u^{b_{2}+l_{2}+l_{3}-1} e^{-u} \mathrm{~d} u .
\end{aligned}
$$

Using these above equations in (2.2), we obtain

$$
\begin{aligned}
& H_{B}\left[(\sigma ; p, v), b_{1}, b_{2} ; c_{1}, c_{2}, c_{3} ; \theta_{1}, \theta_{2}, \theta_{3}\right] \\
& =\frac{\sum_{l_{1}, l_{2}, l_{3}=0}^{\infty}(\sigma ; p, v)_{l_{1}+l_{3}}\left(b_{1}\right)_{l_{1}+l_{2}}\left(b_{2}\right) l_{2}+l_{3}}{\left(c_{1}\right)_{l}\left(c_{2}\right) l_{2}\left(c_{3}\right)_{l_{3}}} \frac{\theta_{1}^{l_{1}}}{l_{1} !} \frac{\theta_{2}^{l_{2}}}{l_{2} !} \frac{\theta_{3}^{l_{3}}}{l_{3} !} \\
& =\frac{\sum_{l_{1}, l_{2}, l_{3}=0}^{\infty} \sqrt{\frac{2 p}{\pi}} \frac{1}{\Gamma(\sigma)} \int_{0}^{\infty} t^{\sigma+l_{1}+l_{3}-\frac{3}{2}} e^{-t} K_{v+\frac{1}{2}}\left(\frac{p}{t}\right) \mathrm{d} t \frac{1}{\Gamma\left(b_{1}\right)} \int_{0}^{\infty} s^{b_{1}+l_{1}+l_{2}-1} e^{-s} \mathrm{~d} s \frac{1}{\Gamma\left(b_{2}\right)} \int_{0}^{\infty} u^{b_{2}+l_{2}+l_{3}-1} e^{-u} \mathrm{~d} u}{\left(c_{1}\right)_{l}\left(c_{2}\right) l_{2}\left(c_{3}\right) l_{3}} \\
& \times \frac{\theta_{1}^{l_{1}}}{l_{1} !} \frac{\theta_{2}^{l_{2}}}{l_{2} !} \frac{\theta_{3}^{l_{3}}}{l_{3} !} \\
& =\frac{\sqrt{\frac{2 p}{\pi}}}{\Gamma(\sigma) \Gamma\left(b_{1}\right) \Gamma\left(b_{2}\right)} \int_{0}^{\infty} \int_{0}^{\infty} \int_{0}^{\infty} t^{\sigma+l_{1}+l_{3}-\frac{3}{2}} s^{b_{1}+l_{1}+l_{2}-1} u^{b_{2}+l_{2}+l_{3}-1} e^{-(s+t+u)} K_{v+\frac{1}{2}}\left(\frac{p}{t}\right) \\
& \times \sum_{l_{1}, l_{2}, l_{3}=0}^{\infty} \frac{1}{\left(c_{1}\right)_{l}\left(c_{2}\right)_{l_{2}}\left(c_{3}\right)_{l_{3}}} \frac{\theta_{1}^{l_{1}}}{l_{1} !} \frac{\theta_{2}^{l_{2}}}{l_{2} !} \frac{\theta_{3}^{l_{3}}}{l_{3} !} \mathrm{d} s \mathrm{~d} t \mathrm{~d} u .
\end{aligned}
$$

Hence

$$
\begin{aligned}
& H_{B}\left[(\sigma ; p, v), b_{1}, b_{2} ; c_{1}, c_{2} ; c_{3} ; \theta_{1}, \theta_{2}, \theta_{3}\right] \\
& =\frac{\sqrt{\frac{2 p}{\pi}}}{\Gamma(\sigma) \Gamma\left(b_{1}\right) \Gamma\left(b_{2}\right)} \int_{0}^{\infty} \int_{0}^{\infty} \int_{0}^{\infty} t^{\sigma+l_{1}+l_{3}-\frac{3}{2}} s^{b_{1}+l_{1}+l_{2}-1} u^{b_{2}+l_{2}+l_{3}-1} e^{-(s+t+u)} K_{v+\frac{1}{2}}\left(\frac{p}{t}\right){ }_{0} F_{1}\left(-; c_{1} ; \theta_{1} s t\right) \\
& \quad \times{ }_{0} F_{1}\left(-; c_{2} ; \theta_{2} u s\right)_{0} F_{1}\left(-; c_{3} ; \theta_{3} u t\right) \mathrm{d} s \mathrm{~d} t \mathrm{~d} u,
\end{aligned}
$$

as desired. 
Theorem 3.4. For $\mathbb{R}(p)>0, \mathbb{R}\left(\theta_{2}\right)<1, \mathbb{R}\left(\theta_{3}\right)<1, \mathbb{R}(\sigma)>0$ and $\mathbb{R}\left(b_{1}\right)>0$, the integral

$$
\begin{aligned}
& H_{C}\left[(\sigma ; p, v), b_{1}, b_{2} ; \gamma ; \theta_{1}, \theta_{2}, \theta_{3}\right] \\
& \quad=\frac{\sqrt{\frac{2 p}{\pi}}}{\Gamma(\sigma) \Gamma\left(b_{1}\right)} \int_{0}^{\infty} \int_{0}^{\infty} t^{\sigma+l_{1}+l_{3}-\frac{3}{2}} s^{b_{1}+l_{1}+l_{2}-1} e^{-(t+s)} K_{v+\frac{1}{2}}\left(\frac{p}{t}\right) \phi_{3}\left(b_{2} ; \gamma ; \theta_{2} s+\theta_{3} t, \theta_{1} s t\right) \mathrm{d} t \mathrm{~d} s
\end{aligned}
$$

holds for the Srivastava hypergeometric function $H_{C}$ defined in (2.3).

Proof. We have

$$
(\sigma ; p, v)_{l_{1}+l_{3}}=\sqrt{\frac{2 p}{\pi}} \frac{1}{\Gamma(\sigma)} \int_{0}^{\infty} t^{\sigma+l_{1}+l_{3}-\frac{3}{2}} e^{-t} K_{v+\frac{1}{2}}\left(\frac{p}{t}\right) \mathrm{d} t
$$

and

$$
\left(b_{1}\right)_{l_{1}+l_{2}}=\frac{1}{\Gamma\left(b_{1}\right)} \int_{0}^{\infty} s^{b_{1}+l_{1}+l_{2}-1} e^{-s} \mathrm{~d} s .
$$

Using these both equations in (2.3), we obtain

$$
\begin{aligned}
& H_{C}\left[(\sigma ; p, v), b_{1}, b_{2} ; \gamma ; \theta_{1}, \theta_{2}, \theta_{3}\right] \\
& =\frac{\sum_{l_{1}, l_{2}, l_{3}=0}^{\infty}(\sigma ; p, v)_{l_{1}+l_{3}}\left(b_{1}\right)_{l_{1}+l_{2}}\left(b_{2}\right)_{l_{2}+l_{3}}}{(y)_{l_{1}+l_{2}+l_{3}}} \frac{\theta_{1}^{l_{1}}}{l_{1} !} \frac{\theta_{2}^{l_{2}}}{l_{2} !} \frac{\theta_{3}^{l_{3}}}{l_{3} !} \\
& =\frac{\sum_{l_{1}, l_{2}, l_{3}=0}^{\infty} \sqrt{\frac{2 p}{\pi}} \frac{1}{\Gamma(\sigma)} \int_{0}^{\infty} t^{\sigma+l_{1}+l_{3}-\frac{3}{2}} e^{-t} K_{v+\frac{1}{2}}\left(\frac{p}{t}\right) \mathrm{d} t \frac{1}{\Gamma\left(b_{1}\right)} \int_{0}^{\infty} s^{b_{1}+l_{1}+l_{2}-1} e^{-s} \mathrm{~d} s\left(b_{2}\right) l_{2}+l_{3}}{(\gamma)_{l_{1}+l_{2}+l_{3}}} \frac{\theta_{1}^{l_{1}}}{l_{1} !} \frac{\theta_{2}^{l_{2}}}{l_{2} !} \frac{\theta_{3}^{l_{3}}}{l_{3} !} \\
& =\sqrt{\frac{2 p}{\pi}} \frac{1}{\Gamma(\sigma)} \int_{0}^{\infty} t^{\sigma+l_{1}+l_{3}-\frac{3}{2}} e^{-t} K_{v+\frac{1}{2}}\left(\frac{p}{t}\right) \mathrm{d} t \frac{1}{\Gamma\left(b_{1}\right)} \int_{0}^{\infty} s^{b_{1}+l_{1}+l_{2}-1} e^{-s} \mathrm{~d} s \sum_{l_{1}, l_{2}, l_{3}=0}^{\infty} \frac{\left(b_{2}\right)_{l_{2}+l_{3}}}{(\gamma)_{l_{1}+l_{2}+l_{3}}} \frac{\theta_{1}^{l_{1}}}{l_{1} !} \frac{\theta_{2}^{l_{2}}}{l_{2} !} \frac{\theta_{3}^{l_{3}}}{l_{3} !} \\
& =\frac{\sqrt{\frac{2 p}{\pi}}}{\Gamma(\sigma) \Gamma\left(b_{1}\right)} \int_{0}^{\infty} \int_{0}^{\infty} t^{\sigma+l_{1}+l_{3}-\frac{3}{2}} s^{b_{1}+l_{1}+l_{2}-1} e^{-(t+s)} K_{v+\frac{1}{2}}\left(\frac{p}{t}\right) \mathrm{d} t \mathrm{~d} s \sum_{l_{1}, l_{2}, l_{3}=0}^{\infty} \frac{\left(b_{2}\right)_{l_{2}+l_{3}}}{(\gamma)_{l_{1}+l_{2}+l_{3}}} \frac{\theta_{1}^{l_{1}}}{l_{1} !} \frac{\theta_{2}^{l_{2}}}{l_{2} !} \frac{\theta_{3}^{l_{3}}}{l_{3} !} .
\end{aligned}
$$

Hence

$$
\begin{aligned}
& H_{C}\left[(\sigma ; p, v), b_{1}, b_{2} ; \gamma ; \theta_{1}, \theta_{2}, \theta_{3}\right] \\
& \quad=\frac{\sqrt{\frac{2 p}{\pi}}}{\Gamma(\sigma) \Gamma\left(b_{1}\right)} \int_{0}^{\infty} \int_{0}^{\infty} t^{\sigma+l_{1}+l_{3}-\frac{3}{2}} s^{b_{1}+l_{1}+l_{2}-1} e^{-(t+s)} K_{v+\frac{1}{2}}\left(\frac{p}{t}\right) \phi_{3}\left(b_{2} ; \gamma ; \theta_{2} s+\theta_{3} t, \theta_{1} s t\right) \mathrm{d} t \mathrm{~d} s .
\end{aligned}
$$

By using the identity in (1.2), we obtain

$$
\phi_{3}\left[\sigma ; c ; \theta_{1}, \theta_{2}\right]=\sum_{m, n=0}^{\infty} \frac{(\sigma)_{l_{2}}}{(c)_{l_{2}+l_{3}}} \frac{\theta_{1}^{l_{2}}}{l_{2} !} \frac{\theta_{2}^{l_{3}}}{l_{3} !}
$$

Theorem 3.5. For $\mathbb{R}(p)>0$ and $\min \left\{\mathbb{R}(\sigma), \mathbb{R}\left(b_{1}\right), \mathbb{R}\left(b_{2}\right)\right\}>0$, the triple integral

$$
\begin{aligned}
H_{C}\left[(\sigma ; p, v), b_{1}, b_{2} ; \gamma ; \theta_{1}, \theta_{2}, \theta_{3}\right]=\frac{\sqrt{\frac{2 p}{\pi}}}{\Gamma(\sigma) \Gamma\left(b_{1}\right) \Gamma\left(b_{2}\right)} \int_{0}^{\infty} \int_{0}^{\infty} \int_{0}^{\infty} t^{\sigma+l_{1}+l_{3}-\frac{3}{2}} s^{b_{1}+l_{1}+l_{2}-1} u^{b_{2}+l_{2}+l_{3}-1} e^{-(t+s+u)} \\
\times K_{v+\frac{1}{2}}\left(\frac{p}{t}\right)_{0} F_{1}\left(-; \gamma ; \theta_{1} s t+\theta_{2} u s+\theta_{3} u t\right) \mathrm{d} t \mathrm{~d} s \mathrm{~d} u
\end{aligned}
$$

holds for the Srivastava hypergeometric function $H_{C}$ defined in (2.3). 
Proof. We have

$$
\begin{aligned}
(\sigma ; p, v)_{l_{1}+l_{3}} & =\sqrt{\frac{2 p}{\pi}} \frac{1}{\Gamma(\sigma)} \int_{0}^{\infty} t^{\sigma+l_{1}+l_{3}-\frac{3}{2}} e^{-t} K_{v+\frac{1}{2}}\left(\frac{p}{t}\right) \mathrm{d} t \\
\left(b_{1}\right)_{l_{1}+l_{2}} & =\frac{1}{\Gamma\left(b_{1}\right)} \int_{0}^{\infty} s^{b_{1}+l_{1}+l_{2}-1} e^{-s} \mathrm{~d} s \\
\left(b_{2}\right) l_{2}+l_{3} & =\frac{1}{\Gamma\left(b_{2}\right)} \int_{0}^{\infty} u^{b_{2}+l_{2}+l_{3}-1} e^{-u} \mathrm{~d} u .
\end{aligned}
$$

Using these three equations in (2.3), we obtain

$$
\begin{aligned}
& H_{C}\left[(\sigma ; p, v), b_{1}, b_{2} ; \gamma ; \theta_{1}, \theta_{2}, \theta_{3}\right] \\
& =\frac{\sum_{l_{1}, l_{2}, l_{3}=0}^{\infty}(\sigma ; p, v)_{l_{1}+l_{3}}\left(b_{1}\right) l_{l_{1}+l_{2}}\left(b_{2}\right) l_{l_{2}+l_{3}}}{(y)_{l_{1}+l_{2}+l_{3}}} \frac{\theta_{1}^{l_{1}}}{l_{1} !} \frac{\theta_{2}^{l_{2}}}{l_{2} !} \frac{\theta_{3}^{l_{3}}}{l_{3} !} \\
& =\frac{\sum_{l_{1}, l_{2}, l_{3}=0}^{\infty} \sqrt{\frac{2 p}{\pi}} \frac{1}{\Gamma(\sigma)} \int_{0}^{\infty} t^{\sigma+l_{1}+l_{3}-\frac{3}{2}} e^{-t} K_{v+\frac{1}{2}}\left(\frac{p}{t}\right) \mathrm{d} t \frac{1}{\Gamma\left(b_{1}\right)} \int_{0}^{\infty} s^{b_{1}+l_{1}+l_{2}-1} e^{-s} \mathrm{~d} s \frac{1}{\Gamma\left(b_{2}\right)} \int_{0}^{\infty} u^{b_{2}+l_{2}+l_{3}-1} e^{-u} \mathrm{~d} u}{(\gamma)_{l_{1}+l_{2}+l_{3}}} \\
& \times \frac{\theta_{1}^{l_{1}}}{l_{1} !} \frac{\theta_{2}^{l_{2}}}{l_{2} !} \frac{\theta_{3}^{l_{3}}}{l_{3} !} \\
& =\frac{\sqrt{\frac{2 p}{\pi}}}{\Gamma(\sigma) \Gamma\left(b_{1}\right) \Gamma\left(b_{2}\right)} \int_{0}^{\infty} \int_{0}^{\infty} \int_{0}^{\infty} t^{\sigma+l_{1}+l_{3}-\frac{3}{2}} s^{b_{1}+l_{1}+l_{2}-1} u^{b_{2}+l_{2}+l_{3}-1} e^{-(s+t+u)} K_{v+\frac{1}{2}}\left(\frac{p}{t}\right) \sum_{l_{1}, l_{2}, l_{3}=0}^{\infty} \frac{1}{(\gamma)_{l_{1}+l_{2}+l_{3}}} \\
& \times \frac{\theta_{1}^{l_{1}}}{l_{1} !} \frac{\theta_{2}^{l_{2}}}{l_{2} !} \frac{\theta_{3}^{l_{3}}}{l_{3} !} \mathrm{d} s \mathrm{~d} t \mathrm{~d} u
\end{aligned}
$$

Hence

$$
\begin{aligned}
H_{C}\left[(\sigma ; p, v), b_{1}, b_{2} ; \gamma ; \theta_{1}, \theta_{2}, \theta_{3}\right]=\frac{\sqrt{\frac{2 p}{\pi}}}{\Gamma(\sigma) \Gamma\left(b_{1}\right) \Gamma\left(b_{2}\right)} \int_{0}^{\infty} \int_{0}^{\infty} \int_{0}^{\infty} t^{\sigma+l_{1}+l_{3}-\frac{3}{2}} s^{b_{1}+l_{1}+l_{2}-1} u^{b_{2}+l_{2}+l_{3}-1} \\
\quad \times e^{-(t+s+u)} K_{v+\frac{1}{2}}\left(\frac{p}{t}\right){ }_{0} F_{1}\left(-; \gamma ; \theta_{1} s t+\theta_{2} u s+\theta_{3} u t\right) \mathrm{d} t \mathrm{~d} s \mathrm{~d} u,
\end{aligned}
$$

as desired.

Next, we consider the Laguerre polynomial $L_{n}^{(\sigma)}(x)$ (see [15]) of order (index) $a$ and degree $n$ in $x$, the Whittaker function $M_{k, m, n}(x, y)$ of two variables [22], the Bessel function $J_{v}(z)$ and the modified Bessel function $I_{v}(z)$ (see $[9,10,12,15])$, which can be represented in terms of the confluent function $\psi_{2}$ and the confluent hypergeometric functions ${ }_{0} F_{1}$, respectively given by

$$
\begin{aligned}
L_{n}^{(\sigma)}(x) & =\frac{(\sigma+1)_{n}}{l_{3} !}{ }_{1} F_{1}(-n ; \sigma+1 ; x), \\
M_{k, m, n}(x, y) & =x^{m+\frac{1}{2}} y^{n+\frac{1}{2}} e^{-\frac{1}{2}(x+y)} \psi_{2}\left(l_{2}+l_{3}-k+1 ; 2 m+1,2 n+1 ; x, y\right), \\
J_{v}(z) & =\frac{\left(\frac{z}{2}\right)^{v}}{\Gamma(v+1)}{ }_{0} F_{1}\left(-; v+1 ;-\frac{1}{4} z^{2}\right) \\
I_{v}(z) & =\frac{\left(\frac{z}{2}\right)^{v}}{\Gamma(v+1)}{ }_{0} F_{1}\left(-; v+1 ; \frac{1}{4} z^{2}\right)
\end{aligned}
$$

The following certain integral representations for the new extension of Srivastava's hypergeometric function $H_{A}$ in (2.1) can be deduced by applying the relations (3.5) to (3.1), (3.7) and (3.8) to (3.1), and (3.5), (3.7) and (3.8) to (3.1), respectively, which are asserted by corollaries. 
Corollary 3.6. The integral

$H_{A}\left[(\sigma ; x ; v), b_{1},-m ; c_{1}, c_{2}+1 ; \theta_{1}, \theta_{2}, \theta_{3}\right]$

$=\frac{\sqrt{\frac{2 x}{\pi}}}{\Gamma(\sigma) \Gamma\left(b_{1}\right)} \frac{l_{2} !}{\left(c_{2}+1\right)_{l_{2}}} \int_{0}^{\infty} \int_{0}^{\infty} t^{\sigma+l_{1}+l_{3}-\frac{3}{2}} s^{b_{1}+l_{1}+l_{2}-1} e^{-(t+s)} K_{v+\frac{1}{2}}\left(\frac{p}{t}\right){ }_{0} F_{1}\left(-; c_{1} ; \theta_{1} s t\right) L_{m}^{\left(c_{2}\right)}\left(\theta_{2} s+\theta_{3} t\right) \mathrm{d} t \mathrm{~d} s$

holds for $H_{A}$ in (2.1) such that the integral involved is convergent.

Corollary 3.7. The integrals

$$
\begin{aligned}
H_{A}\left[(\sigma ; p, v), b_{1}, b_{2} ; c_{1}+1, c_{2} ;-\theta_{1}, \theta_{2}, \theta_{3}\right]=\sqrt{\frac{2 p}{\pi}} & \frac{\Gamma\left(c_{1}+1\right)}{\Gamma(\sigma) \Gamma\left(b_{1}\right)} \theta_{1}^{-\frac{c_{1}}{2}} \int_{0}^{\infty} \int_{0}^{\infty} t^{\sigma+l_{1}+l_{3}-\frac{c_{1}}{2}-\frac{3}{2}} s^{b_{1}+l_{1}+l_{2}-\frac{c_{1}}{2}-1} \\
& \times e^{-(t+s)} K_{v+\frac{1}{2}}\left(\frac{p}{t}\right) J_{c_{1}}\left(2 \sqrt{\theta_{1} s t}\right){ }_{1} F_{1}\left(b_{2} ; c_{2} ; \theta_{2} s+\theta_{3} t\right) \mathrm{d} t \mathrm{~d} s
\end{aligned}
$$

and

$$
\begin{aligned}
H_{A}\left[(\sigma ; p, v), b_{1}, b_{2} ; c_{1}+1, c_{2} ; \theta_{1}, \theta_{2}, \theta_{3}\right]=\sqrt{\frac{2 p}{\pi}} \frac{\Gamma\left(c_{1}+1\right)}{\Gamma(\sigma) \Gamma\left(b_{1}\right)} \theta_{1}^{-\frac{c_{1}}{2}} \int_{0}^{\infty} \int_{0}^{\infty} t^{\sigma+l_{1}+l_{3}-\frac{c_{1}}{2}-\frac{3}{2}} s^{b_{1}+l_{1}+l_{2}-\frac{c_{1}}{2}-1} \\
\times e^{-(t+s)} K_{v+\frac{1}{2}}\left(\frac{p}{t}\right) I_{c_{1}}\left(2 \sqrt{\theta_{1} s t}\right)_{1} F_{1}\left(b_{2} ; c_{2} ; \theta_{2} s+\theta_{3} t\right) \mathrm{d} t \mathrm{~d} s
\end{aligned}
$$

hold for $H_{A}$ in (2.1) such that the integrals involved are convergent.

Corollary 3.8. The integrals

$$
\begin{aligned}
& H_{A}\left[(\sigma ; p, v), b_{1},-m, c_{1}+1, c_{2}+1 ;-\theta_{1}, \theta_{2}, \theta_{3}\right] \\
& =\frac{\sqrt{\frac{2 x}{\pi}}}{\Gamma(\sigma) \Gamma\left(b_{1}\right)} \frac{l_{2} !}{\left(c_{2}+1\right)_{l_{2}}} \Gamma\left(c_{1}+1\right) \theta_{1}^{-\frac{c_{1}}{2}} \int_{0}^{\infty} \int_{0}^{\infty} t^{\sigma+l_{1}+l_{3}-\frac{c_{1}}{2}-\frac{3}{2}} \\
& \quad \times s^{b_{1}+l_{1}+l_{2}-\frac{c_{1}}{2}-1} e^{-(t+s)} K_{v+\frac{1}{2}}\left(\frac{p}{t}\right) J_{c_{1}}\left(2 \sqrt{\left.\theta_{1} s t\right)} L_{m}^{\left(c_{2}\right)}\left(\theta_{2} s+\theta_{3} t\right) \mathrm{d} t \mathrm{~d} s\right.
\end{aligned}
$$

and

$$
\begin{aligned}
& H_{A}\left[(\sigma ; p, v), b_{1},-m, c_{1}+1, c_{2}+1 ; \theta_{1}, \theta_{2}, \theta_{3}\right] \\
& =\frac{\sqrt{\frac{2 x}{\pi}}}{\Gamma(\sigma) \Gamma\left(b_{1}\right)} \frac{l_{2} !}{\left(c_{2}+1\right)_{l_{2}}} \Gamma\left(c_{1}+1\right) \theta_{1}^{-\frac{c_{1}}{2}} \int_{0}^{\infty} \int_{0}^{\infty} t^{\sigma+l_{1}+l_{3}-\frac{c_{1}}{2}-\frac{3}{2}} \\
& \quad \times s^{b_{1}+l_{1}+l_{2}-\frac{c_{1}}{2}-1} e^{-(t+s)} K_{v+\frac{1}{2}}\left(\frac{p}{t}\right) I_{c_{1}}\left(2 \sqrt{\theta_{1} s t}\right) L_{m}^{\left(c_{2}\right)}\left(\theta_{2} s+\theta_{3} t\right) \mathrm{d} t \mathrm{~d} s
\end{aligned}
$$

hold for $H_{A}$ in (2.1) such that the integrals involved are convergent.

Further, we deduce the following certain integral representations for the new extension of Srivastava's hypergeometric function $H_{B}$ in (2.2) by applying the relations (3.6) to (3.2), (3.7) and (3.8) to (3.2), (3.6), (3.7) and (3.8) to (3.2), and (3.7) and (3.8) to (3.3), respectively, which are asserted by corollaries.

Corollary 3.9. The integrals

$$
\begin{aligned}
& H_{B}\left[(\sigma ; p, v), b_{1}, b_{2} ; c_{1}+1, c_{2} ; c_{3} ;-\theta_{1}, \theta_{2}, \theta_{3}\right] \\
& =\sqrt{\frac{2 p}{\pi}} \frac{\Gamma\left(c_{1}+1\right)}{\Gamma(\sigma) \Gamma\left(b_{1}\right)} \theta_{1}^{-\frac{c_{1}}{2}} \int_{0}^{\infty} \int_{0}^{\infty} t^{\sigma+l_{1}+l_{3}-\frac{c_{1}}{2}-\frac{3}{2}} s^{b_{1}+l_{1}+l_{2}-\frac{c_{1}}{2}-1} \\
& \quad \times e^{-(t+s)} K_{v+\frac{1}{2}}\left(\frac{p}{t}\right) J_{c_{1}}\left(2 \sqrt{\theta_{1} s t}\right) \psi_{2}\left(b_{2} ; c_{2}, c_{3} ; \theta_{2} s, \theta_{3} t\right) \mathrm{d} t \mathrm{~d} s
\end{aligned}
$$


and

$$
\begin{aligned}
& H_{B}\left[(\sigma ; p, v), b_{1}, b_{2} ; c_{1}+1, c_{2} ; \theta_{1}, \theta_{2}, \theta_{3}\right] \\
& =\sqrt{\frac{2 p}{\pi}} \frac{\Gamma\left(c_{1}+1\right)}{\Gamma(\sigma) \Gamma\left(b_{1}\right)} \theta_{1}^{-\frac{c_{1}}{2}} \int_{0}^{\infty} \int_{0}^{\infty} t^{\sigma+l_{1}+l_{3}-\frac{c_{1}}{2}-\frac{3}{2}} s^{b_{1}+l_{1}+l_{2}-\frac{c_{1}}{2}-1} \\
& \quad \times e^{-(t+s)} K_{v+\frac{1}{2}}\left(\frac{p}{t}\right) I_{c_{1}}\left(2 \sqrt{\theta_{1} s t}\right) \psi_{2}\left(b_{2} ; c_{2}, c_{3} ; \theta_{2} s, \theta_{3} t\right) \mathrm{d} t \mathrm{~d} s
\end{aligned}
$$

hold for $H_{B}$ in (2.2) such that the integrals involved are convergent.

Corollary 3.10. The integral

$$
\begin{aligned}
& H_{B}\left[(\sigma ; p, v), \mu, \sigma+p-k+1 ; z, 2 \sigma+1,2 p+1 ; \theta_{1}, \theta_{2}, \theta_{3}\right] \\
& =\frac{\sqrt{\frac{2 p}{\pi}}}{\Gamma(\sigma) \Gamma(\mu)} \theta_{2}^{-\sigma-\frac{1}{2}} \theta_{3}^{-p-\frac{1}{2}} \int_{0}^{\infty} \int_{0}^{\infty} t^{\sigma+l_{1}+l_{3}-p-\frac{3}{2}} s^{\mu+l_{1}+l_{2}-\sigma-\frac{3}{2}} e^{-\left(1-\frac{1}{2} \theta_{2}\right) s-\left(1-\frac{1}{2} \theta_{3}\right) t} K_{v+\frac{1}{2}}\left(\frac{p}{t}\right) \\
& \quad \times{ }_{0} F_{1}\left(-; z ; \theta_{1} s t\right) M_{k, \sigma, p}\left(\theta_{2} s, \theta_{3} t\right) \mathrm{d} t \mathrm{~d} s
\end{aligned}
$$

holds for $H_{B}$ in (2.2) such that given integral is convergent.

Corollary 3.11. The double integrals

$$
\begin{aligned}
& H_{B}\left[(\sigma ; p, v), \mu, \sigma+p-k+1 ; z+1,2 \sigma+1,2 p+1 ;-\theta_{1}, \theta_{2}, \theta_{3}\right] \\
& =\frac{\sqrt{\frac{2 p}{\pi}}}{\Gamma(\sigma) \Gamma(\mu)} \theta_{1}^{-\frac{1}{2} z} \theta_{2}^{-\sigma-\frac{1}{2}} \theta_{3}^{-p-\frac{1}{2}} \int_{0}^{\infty} \int_{0}^{\infty} t^{\sigma+l_{1}+l_{3}-p-\frac{1}{2} z-\frac{3}{2}} s^{\mu+l_{1}+l_{2}-\sigma-\frac{1}{2} z-\frac{3}{2}} \\
& \quad \times e^{-\left(1-\frac{1}{2} \theta_{2}\right) s-\left(1-\frac{1}{2} \theta_{3}\right) t} K_{v+\frac{1}{2}}\left(\frac{p}{t}\right) J\left(2 \sqrt{\theta_{1} s t}\right) M_{k, \sigma, p}\left(\theta_{2} s, \theta_{3} t\right) \mathrm{d} t \mathrm{~d} s
\end{aligned}
$$

and

$$
\begin{aligned}
& H_{B}\left[(\sigma ; p, v), \mu, \sigma+p-k+1 ; z+1,2 \sigma+1,2 p+1 ; \theta_{1}, \theta_{2}, \theta_{3}\right] \\
& =\frac{\sqrt{\frac{2 p}{\pi}}}{\Gamma(\sigma) \Gamma(\mu)} \theta_{1}^{-\frac{1}{2} z} \theta_{2}^{-\sigma-\frac{1}{2}} \theta_{3}^{-p-\frac{1}{2}} \int_{0}^{\infty} \int_{0}^{\infty} t^{\sigma+l_{1}+l_{3}-p-\frac{1}{2} z-\frac{3}{2}} s^{\mu+l_{1}+l_{2}-\sigma-\frac{1}{2} z-\frac{3}{2}} \\
& \quad \times e^{-\left(1-\frac{1}{2} \theta_{2}\right) s-\left(1-\frac{1}{2} \theta_{3}\right) t} K_{v+\frac{1}{2}}\left(\frac{p}{t}\right) I\left(2 \sqrt{\theta_{1} s t}\right) M_{k, \sigma, p}\left(\theta_{2} s, \theta_{3} t\right) \mathrm{d} t \mathrm{~d} s
\end{aligned}
$$

hold for $H_{B}$ in (2.2) such that the integrals involved are convergent.

Corollary 3.12. The triple integrals

$$
\begin{gathered}
H_{B}\left[(\sigma ; p, v), b_{1}, b_{2} ; c_{1}+1, c_{2}+1, c_{3}+1 ;-\theta_{1},-\theta_{2},-\theta_{3}\right] \\
=\frac{\sqrt{\frac{2 p}{\pi}} \Gamma\left(c_{1}+1\right) \Gamma\left(c_{2}+1\right) \Gamma\left(c_{3}+1\right)}{\Gamma(\sigma) \Gamma\left(b_{1}\right) \Gamma\left(b_{2}\right)} \theta_{1}^{-\frac{c_{1}}{2}} \theta_{2}^{-\frac{c_{2}}{2}} \theta_{3}^{-\frac{c_{3}}{2}} \\
\quad \times \int_{0}^{\infty} \int_{0}^{\infty} \int_{0}^{\infty} t^{\sigma+l_{1}+l_{3}-\frac{c_{1}}{2}-\frac{c_{3}}{2}-\frac{3}{2}} s^{b_{1}+l_{1}+l_{2}-\frac{c_{1}}{2}-\frac{c_{2}}{2}-1} u^{b_{2}+l_{2}+l_{3}-\frac{c_{2}}{2}-\frac{c_{3}}{2}-1} e^{-(s+t+u)} \\
\times K_{v+\frac{1}{2}}\left(\frac{p}{t}\right) J_{c_{1}}\left(2 \sqrt{\theta_{1} s t}\right) J_{c_{2}}\left(2 \sqrt{\theta_{2} u s}\right) J_{c_{3}}\left(2 \sqrt{\theta_{3} u t}\right) \mathrm{d} t \mathrm{~d} s \mathrm{~d} u
\end{gathered}
$$


and

$$
\begin{aligned}
& H_{B}\left[(\sigma ; p, v), b_{1}, b_{2} ; c_{1}+1, c_{2}+1, c_{3}+1 ; \theta_{1}, \theta_{2}, \theta_{3}\right] \\
& =\frac{\sqrt{\frac{2 p}{\pi}} \Gamma\left(c_{1}+1\right) \Gamma\left(c_{2}+1\right) \Gamma\left(c_{3}+1\right)}{\Gamma(\sigma) \Gamma\left(b_{1}\right) \Gamma\left(b_{2}\right)} \theta_{1}^{-\frac{c_{1}}{2}} \theta_{2}^{-\frac{c_{2}}{2}} \theta_{3}^{-\frac{c_{3}}{2}} \\
& \quad \times \int_{0}^{\infty} \int_{0}^{\infty} \int_{0}^{\infty} t^{\sigma+l_{1}+l_{3}-\frac{c_{1}}{2}-\frac{c_{3}}{2}-\frac{3}{2}} s^{b_{1}+l_{1}+l_{2}-\frac{c_{1}}{2}-\frac{c_{2}}{2}-1} u^{b_{2}+l_{2}+l_{3}-\frac{c_{2}}{2}-\frac{c_{3}}{2}-1} e^{-(s+t+u)} \\
& \quad \times K_{v+\frac{1}{2}}\left(\frac{p}{t}\right) I_{c_{1}}\left(2 \sqrt{\theta_{1} s t}\right) I_{c_{2}}\left(2 \sqrt{\theta_{2} u s}\right) I_{c_{3}}\left(2 \sqrt{\theta_{3} u t}\right) \mathrm{d} t \mathrm{~d} s \mathrm{~d} u
\end{aligned}
$$

hold for $H_{B}$ in (2.2) such that the integrals involved are convergent.

\section{Derivatives and recurrence relation formulas}

Certain derivative and recurrence relation formulas for the newly defined functions $H_{A}, H_{B}$ and $H_{C}$ in (2.1), (2.2) and (2.3) are derived in this section.

\subsection{Derivative formulas}

Differentiating both sides of (2.1), (2.2) and (2.3) partially with respect to the variables $\theta_{1}, \theta_{2}$ and $\theta_{3}, l, m$ and $n$ times, respectively, we get the derivative formulas for the functions $H_{A}, H_{B}$ and $H_{C}$ in (2.1),(2.2) and (2.3), which are derived in the following theorem.

Theorem 4.1. The derivative formulas

$$
\begin{aligned}
& \frac{\partial^{l_{1}+l_{2}+l_{3}}}{\partial \theta_{1}^{l_{1}} \partial \theta_{2}^{l_{2}} \partial \theta_{3}^{l_{3}}} H_{A}\left[(\sigma ; p, v), b_{1}, b_{2} ; c_{1}, c_{2} ; \theta_{1}, \theta_{2}, \theta_{3}\right] \\
& =\frac{(\sigma)_{l_{1}+l_{3}}\left(b_{1}\right)_{l_{1}+l_{2}}\left(b_{2}\right)_{l_{2}+l_{3}}}{\left(c_{1}\right)_{l_{1}}\left(c_{2}\right)_{l_{2}+l_{3}}} \\
& \times H_{A}\left[\left(\sigma+l_{1}+l_{3} ; p, v\right), b_{1}+l_{1}+l_{2}, b_{2}+l_{2}+l_{3} ; c_{1}+l, c_{2}+l_{2}+l_{3} ; \theta_{1}, \theta_{2}, \theta_{3}\right], \\
& \frac{\partial^{l_{1}+l_{2}+l_{3}}}{\partial \theta_{1}^{l_{1}} \partial \theta_{2}^{l_{2}} \partial \theta_{3}^{l_{3}}} H_{B}\left[(\sigma ; p, v), b_{1}, b_{2} ; c_{1}, c_{2}, c_{3} ; \theta_{1}, \theta_{2}, \theta_{3}\right] \\
& =\frac{(\sigma)_{l_{1}+l_{3}}\left(b_{1}\right) l_{l_{1}+l_{2}}\left(b_{2}\right)_{l_{2}+l_{3}}}{\left(c_{1}\right)_{l_{1}}\left(c_{2}\right) l_{2}\left(c_{3}\right) l_{3}} \\
& \times H_{B}\left[\left(\sigma+l_{1}+l_{3} ; p, v\right), b_{1}+l_{1}+l_{2}, b_{2}+l_{2}+l_{3} ; c_{1}+l, c_{2}+m, c_{3}+n ; \theta_{1}, \theta_{2}, \theta_{3}\right], \\
& \frac{\partial^{l_{1}+l_{2}+l_{3}}}{\partial \theta_{1}^{l_{1}} \partial \theta_{2}^{l_{2}} \partial \theta_{3}^{l_{3}}} H_{C}\left[(\sigma ; p, v), b_{1}, b_{2} ; \gamma ; \theta_{1}, \theta_{2}, \theta_{3}\right] \\
& =\frac{(\sigma)_{l_{1}+l_{3}}\left(b_{1}\right) l_{1}+l_{2}\left(b_{2}\right) l_{2}+l_{3}}{(y) l_{1}+l_{2}+l_{3}} H_{C}\left[\left(\sigma+l_{1}+l_{3} ; p, v\right), b_{1}+l_{1}+l_{2}, b_{2}+l_{2}+l_{3} ; \gamma+l_{1}+l_{2}+l_{3} ; \theta_{1}, \theta_{2}, \theta_{3}\right]
\end{aligned}
$$

hold for $H_{A}, H_{B}$ and $H_{C}$.

\subsection{Recurrence relations}

We derive recurrence relations for the functions $H_{A}, H_{B}$ and $H_{C}$ in (2.1), (2.2) and (2.3), which are derived in the following theorems. 
Theorem 4.2. The relation

$$
\begin{aligned}
H_{A}\left[(\sigma ; p, v), b_{1}, b_{2} ; c_{1}, c_{2} ; \theta_{1}, \theta_{2}, \theta_{3}\right]=H_{A}\left[(\sigma ; p, v), b_{1}, b_{2} ; c_{1}-1, c_{2} ; \theta_{1}, \theta_{2}, \theta_{3}\right] \\
\quad+\frac{\sigma b_{1} \theta_{1}}{c_{1}\left(1-c_{1}\right)} H_{A}\left[(\sigma+1 ; p, v), b_{1}+1, b_{2} ; c_{1}+1, c_{2} ; \theta_{1}, \theta_{2}, \theta_{3}\right],
\end{aligned}
$$

holds for the function $H_{A}$.

Proof. In the integral representation (3.1), we employ the following relation of the function ${ }_{0} F_{1}$ (see [17]):

$$
{ }_{0} F_{1}(-; y-1 ; x)-{ }_{0} F_{1}(-; \gamma ; x)-\frac{x}{\gamma(y-1)}{ }_{0} F_{1}(-; y+1 ; x)=0 \text {. }
$$

Hence, we get the desired result.

Theorem 4.3. The relation

$$
\begin{aligned}
& H_{B}\left[(\sigma ; p, v), b_{1}, b_{2} ; c_{1}, c_{2}, c_{3} ; \theta_{1}, \theta_{2}, \theta_{3}\right] \\
& =H_{B}\left[(\sigma ; p, v), b_{1}, b_{2} ; c_{1}-1, c_{2}, c_{3} ; \theta_{1}, \theta_{2}, \theta_{3}\right] \\
& +\frac{\sigma b_{1} \theta_{1}}{\gamma(1-\gamma)} H_{B}\left[(\sigma+1 ; p, v), b_{1}+1, b_{2} ; c_{1}+1, c_{2}, c_{3} ; \theta_{1}, \theta_{2}, \theta_{3}\right]
\end{aligned}
$$

holds for the function $H_{B}$.

Proof. Applying the similar procedure used in Theorem 4.2 together with (3.2) will give the desired result.

Theorem 4.4. The relation

$$
\begin{aligned}
H_{C}\left[(\sigma ; p, v), b_{1}, b_{2} ; \gamma ; \theta_{1}, \theta_{2}, \theta_{3}\right]=H_{C}[( & \left.\sigma ; p, v), b_{1}, b_{2} ; \gamma-1 ; \theta_{1}, \theta_{2}, \theta_{3}\right] \\
& +\frac{\sigma b_{1} \theta_{1}}{\gamma(1-\gamma)} H_{C}\left[(\sigma+1 ; p, v), b_{1}+1, b_{2} ; \gamma+1, \gamma+1 ; \theta_{1}, \theta_{2}, \theta_{3}\right] \\
& +\frac{b_{1} b_{2} \theta_{2}}{\gamma(1-\gamma)} H_{C}\left[(\sigma+1 ; p, v), b_{1}+1, b_{2}+1 ; \gamma+1, \gamma+1 ; \theta_{1}, \theta_{2}, \theta_{3}\right] \\
& +\frac{\sigma b_{2} \theta_{3}}{\gamma(1-\gamma)} H_{C}\left[(\sigma+1 ; p, v), b_{1}, b_{2}+1 ; \gamma+1, \gamma+1 ; \theta_{1}, \theta_{2}, \theta_{3}\right]
\end{aligned}
$$

holds for the function $H_{C}$.

Proof. Applying the similar procedure used in Theorem 4.2 together with (3.4) gives the desired result.

\section{Concluding remarks}

In this present paper, we investigated a new extension of the generalized Srivastava's triple hypergeometric functions $H_{A}, H_{B}$ and $H_{C}$ in (2.1), (2.2) and (2.3) by using the extended Pochhammer symbol ( $\left.\varrho ; p, v\right)_{\mu}$ recently defined by Srivastava et al. [29]. Also, we investigated certain special cases when $\theta_{2}=0$ in (2.1), (2.2) and (2.3) are respectively reduced to a new extension of the Appell functions $F_{2}$ and $F_{1}$ of two variables. The special cases of our results by setting $v=0$ can be found in the works [18-22, 25, 26].

\section{References}

[1] M. Arshad, J. Choi, S. Mubeen, K. S. Nisar and G. Rahman, A new extension of the Mittag-Leffler function, Commun. Korean Math. Soc. 33 (2018), no. 2, 549-560.

[2] M. Arshad, S. Mubeen, K. S. Nisar and G. Rahman, Extended Wright-Bessel function and its properties, Commun. Korean Math. Soc. 33 (2018), no. 1, 143-155.

[3] M. A. Chaudhry and S. M. Zubair, Generalized incomplete gamma functions with applications, J. Comput. Appl. Math. 55 (1994), no. 1, 99-124. 
[4] M. A. Chaudhry and S. M. Zubair, On a Class of Incomplete Gamma Functions with Applications, Chapman \& Hall/CRC, Boca Raton, 2002.

[5] J. Choi and R. K. Parmar, Generalized Srivastava's triple hypergeometric functions and their associated properties, J. Nonlinear Sci. Appl. 10 (2017), no. 2, 817-827.

[6] J. Choi, R. K. Parmar and P. Chopra, Extended Mittag-Leffler function and associated fractional calculus operators, Georgian Math. J. 27 (2020), no. 2, 199-209.

[7] J. Choi, R. K. Parmar and T. K. Pogány, Mathieu-type series built by $(p, q)$-extended Gaussian hypergeometric function, Bull. Korean Math. Soc. 54 (2017), no. 3, 789-797.

[8] J. Choi, A. K. Rathie and R. K. Parmar, Extension of extended beta, hypergeometric and confluent hypergeometric functions, Honam Math. J. 36 (2014), no. 2, 357-385.

[9] Y. L. Luke, Mathematical Functions and Their Approximations, Academic Press, New York, 1975.

[10] W. Magnus, F. Oberhettinger and R. P. Soni, Formulas and Theorems for the Special Functions of Mathematical Physics, 3rd enlarged ed., Grundlehren Math. Wiss. 52, Springer, New York, 1966.

[11] S. Mubeen, S. D. Purohit, M. Arshad and G. Rahman, Extension of $k$-gamma, $k$-beta functions and $k$-beta distribution, J. Math. Anal. 7 (2016), no. 5, 118-131.

[12] F. W. J. Olver, D. W. Lozier, R. F. Boisvert and C. W. Clark, NIST Handbook of Mathematical Functions, Cambridge University, Cambridge, 2010.

[13] R. K. Parmar and T. K. Pogány, Extended Srivastava's triple hypergeometric $H_{A, p, q}$ function and related bounding inequalities, Izv. Nats. Akad. Nauk Armenii Mat. 52 (2017), no. 6, 47-61.

[14] T. K. Pogány and R. K. Parmar, On p-extended Mathieu series, Rad Hrvat. Akad. Znan. Umjet. Mat. Znan. 22(534) (2018), 107-117.

[15] E. D. Rainville, Special Functions, Chelsea, New York, 1971.

[16] M. Safdar, G. Rahman, Z. Ullah, A. Ghaffar and K. S. Nisar, A new extension of the Pochhammer symbol and its application to hypergeometric functions, Int. J. Appl. Comput. Math. 5 (2019), no. 6, Paper No. 151.

[17] L. J. Slater, Confluent Hypergeometric Functions, Cambridge University, New York, 1960.

[18] H. M. Srivastava, Hypergeometric functions of three variables, Ganita 15 (1964), 97-108.

[19] H. M. Srivastava, On transformations of certain hypergeometric functions of three variables, Publ. Math. Debrecen 12 (1965), 65-74.

[20] H. M. Srivastava, On the reducibility of certain hypergeometric functions, Univ. Nac. Tucumán Rev. Ser. A 16 (1966), 7-14.

[21] H. M. Srivastava, Relations between functions contiguous to certain hypergeometric functions of three variables, Proc. Nat. Acad. Sci. India Sect. A 36 (1966), 377-385.

[22] H. M. Srivastava, Some integrals representing triple hypergeometric functions, Rend. Circ. Mat. Palermo (2) 16 (1967), 99-115.

[23] H. M. Srivastava, A. Çetinkaya and I. Onur Kıymaz, A certain generalized Pochhammer symbol and its applications to hypergeometric functions, Appl. Math. Comput. 226 (2014), 484-491.

[24] H. M. Srivastava and J. Choi, Zeta and q-Zeta Functions and Associated Series and Integrals, Elsevier, Amsterdam, 2012.

[25] H. M. Srivastava and P. W. Karlsson, Multiple Gaussian Hypergeometric Series, Ellis Horwood Ser. Math. Appl., Ellis Horwood, Chichester, 1985.

[26] H. M. Srivastava and H. L. Manocha, A Treatise on Generating Functions, Ellis Horwood Ser. Math. Appl., Ellis Horwood, Chichester, 1984.

[27] H. M. Srivastava, R. K. Parmar and P. Chopra, A class of extended fractional derivative operators and associated generating relations involving hypergeometric functions, Axioms 1 (2012), 238-258.

[28] H. M. Srivastava, R. K. Parmar and M. M. Joshi, Extended Lauricella and Appell functions and their associated properties, Adv. Stud. Contemp. Math. 25 (2015), 151-165.

[29] H. M. Srivastava, G. Rahman and K. S. Nisar, Some extensions of the Pochhammer symbol and the associated hypergeometric functions, Iran. J. Sci. Technol. Trans. A Sci. 43 (2019), no. 5, 2601-2606.

[30] H. M. Srivastava, A. Tassaddiq, G. Rahman, K. S. Nisar and I. Khan, A new extension of the $\tau$-Gauss hypergeometric function and its associated properties, Mathematics 7 (2019), DOI 10.3390/math7100996. 